\title{
Distribution of a putative endothelial barrier antigen in the ocular and orbital tissues of the rat
}

\author{
J G Lawrenson, M N Ghabriel, A R Reid, T N Gajree, G Allt
}

\section{Reta Lila Weston \\ Institute of \\ Neurological Studies, \\ University College \\ London Medical \\ School, London \\ J G Lawrenson \\ M N Ghabriel \\ A R Reid \\ T N Gajree \\ G Allt}

Applied Vision

Research Centre,

Department of

Science, City

J G Lawrenson

Anatomy and

Histology, University

of Adelaide, South

Australia 5005

M N Ghabriel

Correspondence to: Dr J G Lawrenson, Reta Lila

Weston Institute of

Neurolorit Studies,

University College London

Medical School, Riding

Medical School, Riding

W1P 7PN.

Accepted for publication

9 December 1994
Optometry and Visual

University, London

Department of

\begin{abstract}
Background-A rat endothelial barrier antigen (EBA) recognised by a monoclonal antibody has been shown to be expressed strongly by endothelial cells of brain capillaries possessing a blood-brain barrier and only weakly expressed by fenestrated brain vessels.

Methods-In this study immunocytochemical methods for light and electron microscopy were used to study EBA distribution in the eye and orbital tissues of the rat.

Results-Blood-ocular barrier vessels in the optic nerve, retina, iris, and some vessels in the choroid and ciliary body were immunopositive for EBA. By preembedding immunocytochemistry for electron microscopy the antigen was observed on the luminal endothelial cell surface.

Conclusion-Surprisingly, some nonbarrier vessels in the ciliary body and choroid expressed EBA suggesting that it may play a broader role in endothelial properties than previously recognised. The functional significance of EBA remains to be elucidated.

(Br f Ophthalmol 1995; 79: 462-466)
\end{abstract}

Many ocular blood vessels possess a selective endothelial barrier which is important for

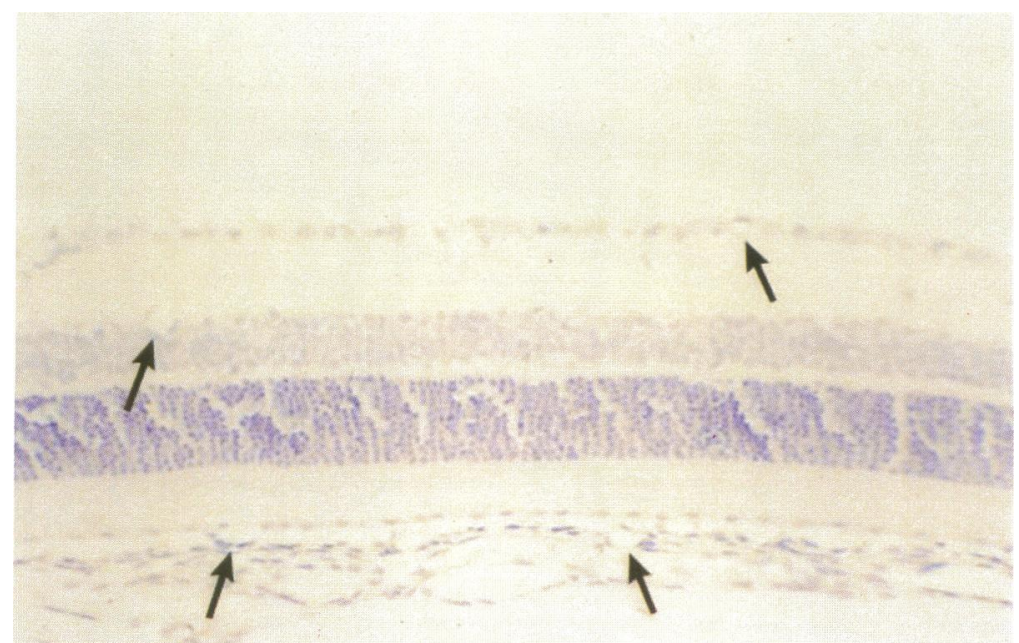

Figure 1 Section of retina (negative control). Microvessels (arrows) in retina and choroid are immunonegative for endothelial barrier antigen. Magnification $\times 131$. maintaining the homeostasis of the internal ocular environment. Structurally, the endothelial cells (EC) of these vessels have many similarities to those found in brain, with multiple tight junctions between adjacent cells and a low density of cytoplasmic vesicular profiles. ${ }^{1}$ The anatomy and physiology of brain EC have been extensively studied ${ }^{2}$ and more recently their molecular biology has received increasing attention. ${ }^{3}$ Over the past 10 years several blood-brain barrier (BBB) specific proteins have been identified, including enzymes and receptor transporter systems. ${ }^{4}$ These molecules, which can be detected by immunocytochemistry at the light and electron microscopic levels using monoclonal or polyclonal antibodies, vary in their degree of BBB specificity and often show an asymmetrical distribution between luminal and abluminal EC plasma membranes. ${ }^{4} \mathrm{~A}$ monoclonal antibody has been described which recognises a protein present in high concentrations on rat brain EC which possess BBB characteristics, and by contrast is low or absent from EC in brain regions lacking a $\mathrm{BBB}$ such as the choroid plexus and area postrema. In view of its association with endothelia at classic $\mathrm{BBB}$ sites the protein has been termed the endothelial barrier antigen (EBA). ${ }^{5}$ The molecular characteristics of EBA are poorly understood. We know from western blots prepared from rat brain microvessels that anti-EBA labels a protein triplet of 30,25 , and $23.5 \mathrm{kDa}$ molecular weight. ${ }^{5}$ However, its function is unknown. The development of EBA immunoreactivity is a postnatal event, occurring during a period 3-20 days post partum. ${ }^{5}$ It thus represents a late phase marker with respect to BBB maturation, in contrast with other barrier specific markers - for example, the erythrocyte/brain (glut-1) isoform of the glucose transporter and the transferrin receptor which are expressed relatively early in the development of the BBB. ${ }^{4}$ Postnatally, EBA immunoreactivity appears to correlate well with an intact BBB. For example, following traumatic brain injury vessels lose their reactivity to anti-EBA for a period which correlates with an increased vascular permeability to serum proteins. ${ }^{6}$

Similarly, altered BBB function in experimental allergic encephalomyelitis (EAE) results in a loss of EBA immunoreactivity for the duration of the active phase of the disease with an increase in EBA labelling on the reestablishment of the BBB. ${ }^{7} \mathrm{~A}$ recent study has 


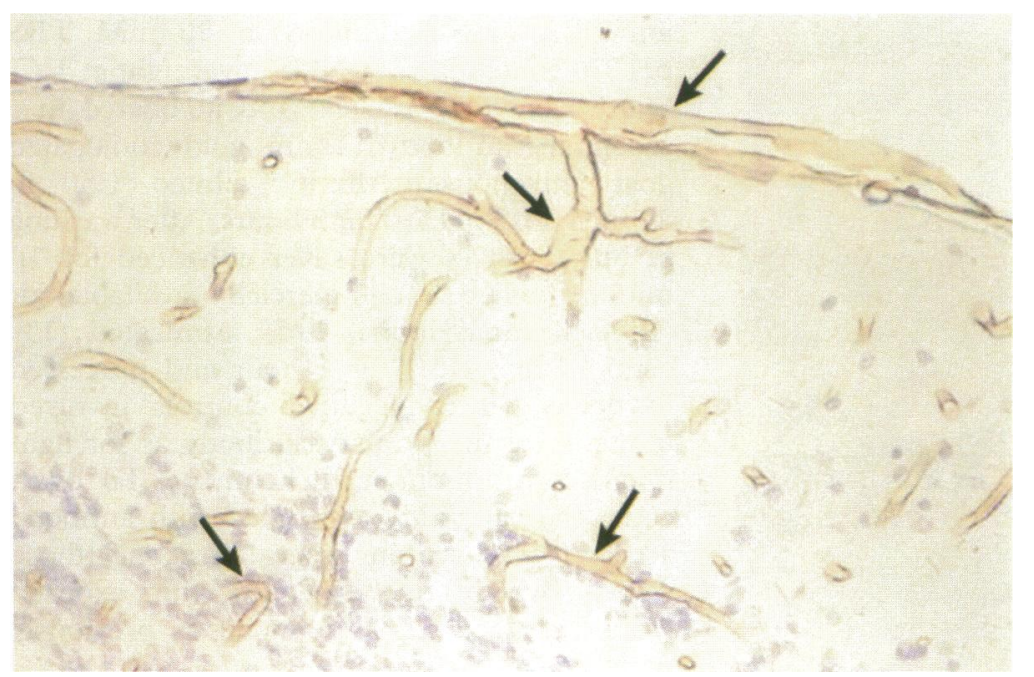

Figure 2 Section of rat brain used as a positive control, showing strongly endothelial barrier antigen (EBA) positive vessels (arrows) in grey and white matter and in the pia mater. Anti-EBA 1:20000. Magnification $\times 193$.

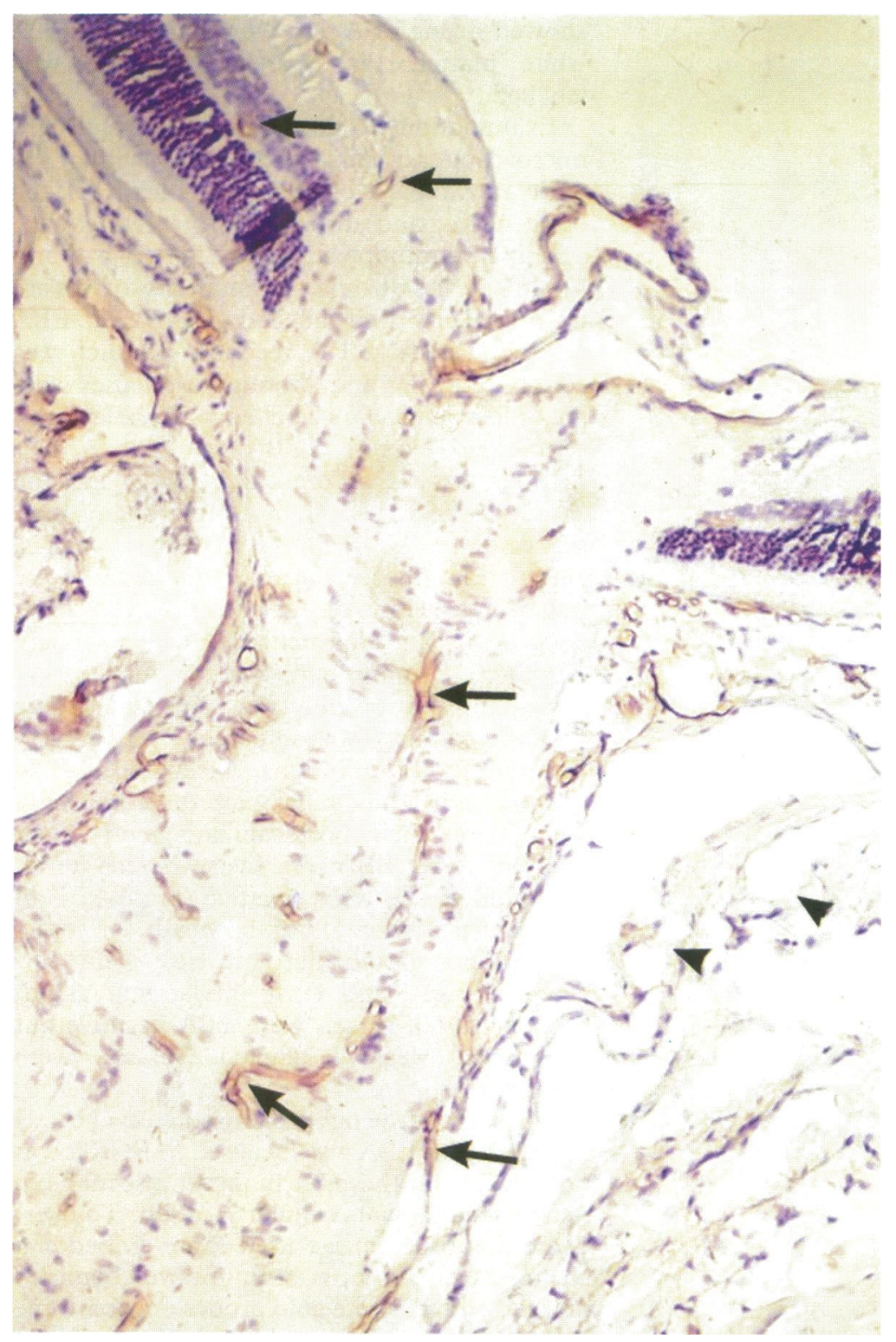

Figure 3 Section through the optic nerve head. Vessels of the optic nerve parenchyma, pia mater, and retina are endothelial barrier antigen (EBA) positive (arrows). Vessels in the dura (arrowheads) are negative. Anti-EBA 1:1000. Magnification $\times 153$. provided some evidence that levels of EBA may also change with age since a reduction in the labelling of hippocampal microvessels was observed in aged rats. ${ }^{8}$

Using anti-EBA we have demonstrated heterogeneity in the expression of the barrier antigen within the central and peripheral nervous system. ${ }^{9}$ The purpose of the present study was to investigate the distribution of EBA in the ocular and orbital tissues of the rat where barrier and non-barrier vessels are known to exist. Using light and electron microscopy immunocytochemistry bloodocular barrier (BOB) vessels were compared with leaky vessels where a BOB is absent.

\section{Materials and methods}

Twelve male Sprague-Dawley rats (200$300 \mathrm{~g})$ were sedated with diazepam $(0 \cdot 2 \mathrm{mg} / \mathrm{kg}$ body weight), anaesthetised with Hypnorm $(0.16 \mathrm{mg} \mathrm{kg}$ body weight), and fixed by perfusion with $2 \%$ paraformaldehyde $/ 0 \cdot 2 \%$ glutaraldehyde in $0.15 \mathrm{M}$ phosphate buffer (pH 7.3). The intraorbital contents including eye and optic nerve, the extraocular muscles, and lacrimal gland were removed and immersed in fresh fixative for 2 hours at $4^{\circ} \mathrm{C}$. Small pieces of tissue were either embedded in paraffin wax for light microscopy immunocytochemistry or in gelatine for vibratome sectioning and subsequent ultrastructural immunocytochemistry.

\section{LIGHT MICROSCOPY IMMUNOCYTOCHEMISTRY}

Paraffin sections $(8-10 \mu \mathrm{m})$ were mounted on coated glass slides (Biobond, BioCell International, Cardiff), dewaxed, and endogenous peroxidase blocked using $0.5 \%$ hydrogen peroxide in absolute methanol for 30 minutes. Sections were then rinsed and covered with $10 \%$ normal goat serum (NGS) for 10 minutes. Incubation in primary antibody (antiEBA monoclonal mouse anti-rat, Affiniti Research Products, Nottingham) diluted $1: 1000-1: 20000$ in $50 \mathrm{mM}$ TRIS buffered saline (TBS) $\mathrm{pH} 7 \cdot 6$ containing $1 \%$ NGS was carried out overnight at $4^{\circ} \mathrm{C}$. After washing in TBS, sections were incubated in a secondary antibody (goat anti-mouse horseradish peroxidase (HRP) conjugate, Affiniti) diluted 1:100 in $20 \mathrm{mM}$ TBS $\mathrm{pH} 7 \cdot 2$ for 3 hours. HRP reaction product was developed by incubating in $0.1 \%$ diaminobenzidine (DAB) and $0.02 \%$ hydrogen peroxide for 2-5 minutes. Sections were washed and lightly counterstained with haematoxylin, dehydrated, and mounted for light microscopy. Negative controls were treated as above with the omission of the primary antibody. As a positive EBA control sections of brain and spinal cord were used.

\section{ELECTRON MICROSCOPY IMMUNOCYTO-} CHEMISTRY

For pre-embedding immunolabelling vibratome slices $(200 \mu \mathrm{m})$ of optic nerve (including its meningeal sheaths) were incubated with 


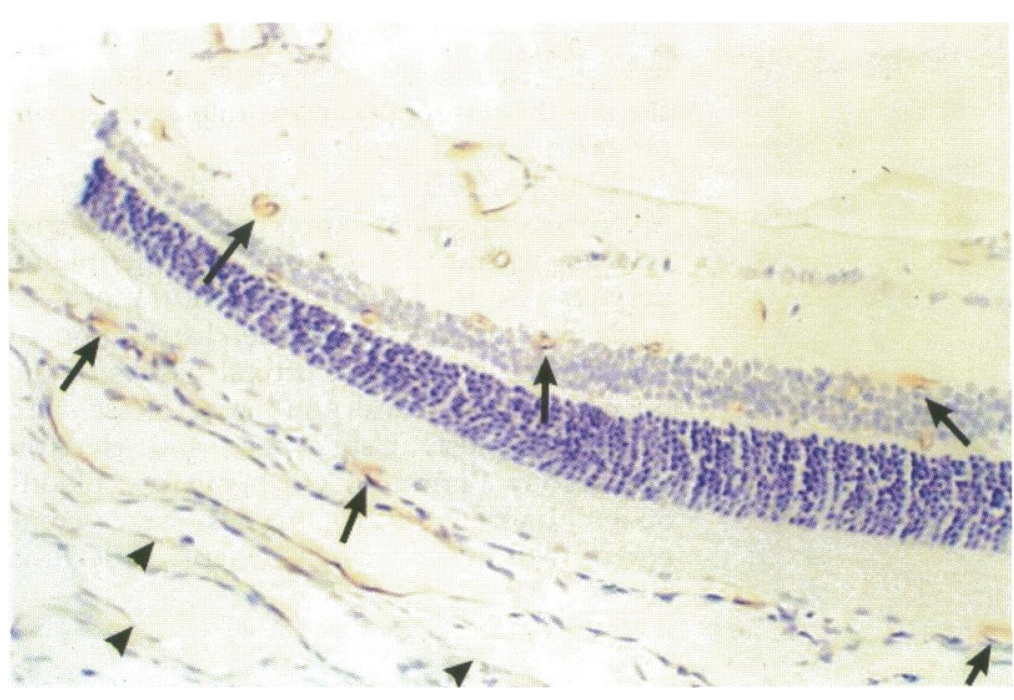

Figure 4 Endothelial barrier antigen (EBA) positive vessels (arrows) in the retina and superficial choroid. Larger choroidal vessels located externally (arrowheads) are negative. Anti-EBA 1:20 000. Magnification $\times 150$.

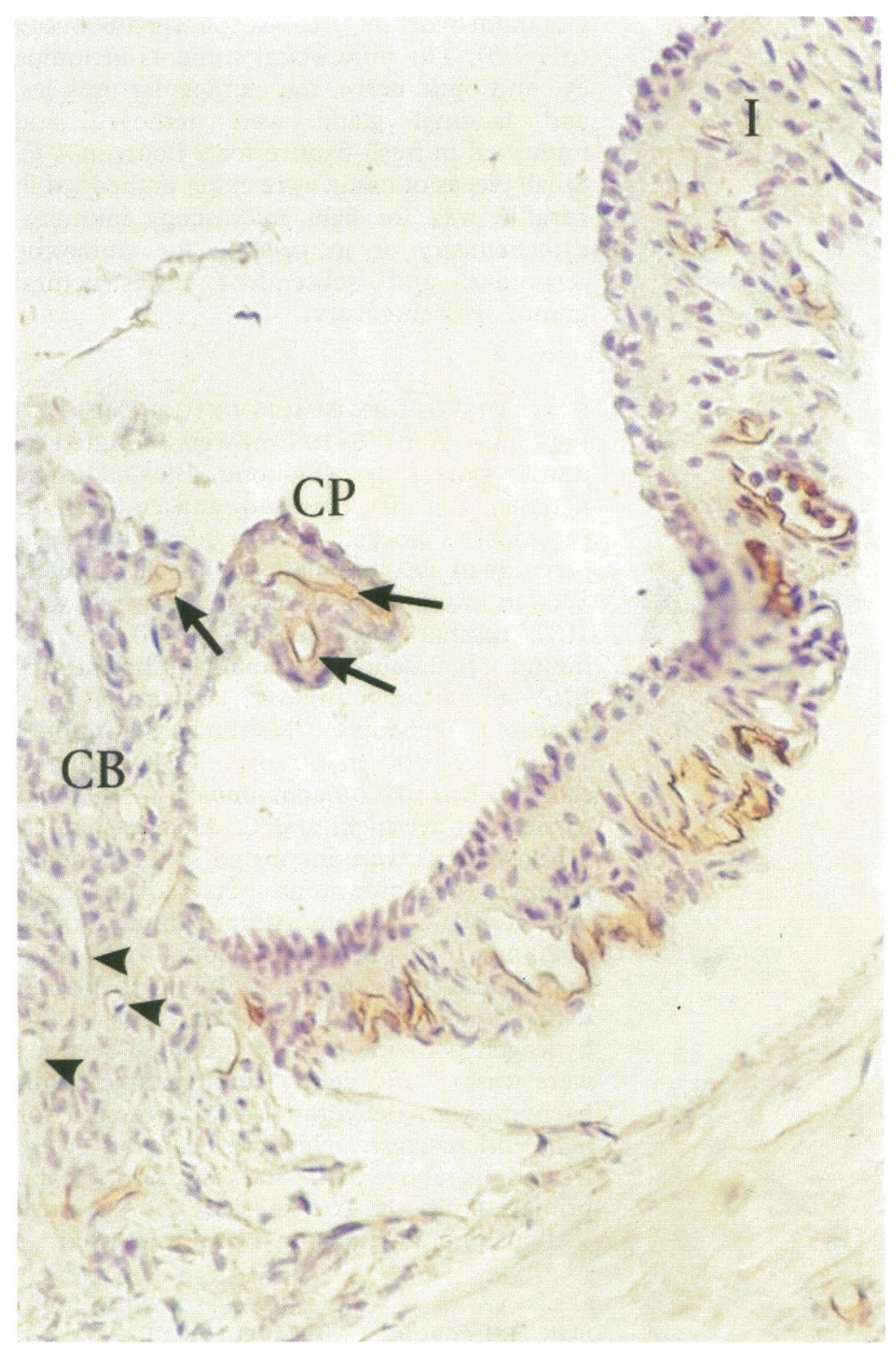

Figure 5 Section of anterior eye segment showing the iris (I) and ciliary body (CB). The majority of iridial vessels are endothelial barrier antigen (EBA) positive. In contrast most vessels in the ciliary body are negative (arrowheads); however, a few positive vessels (arrows) are located close to the ciliary epithelium and particularly within ciliary processes (CP). Anti-EBA 1:1000. Magnification $\times 400$.
anti-EBA (1:100 dilution in $50 \mathrm{mM}$ TBS pH 7.6 containing $1 \%$ NGS) overnight at $4^{\circ} \mathrm{C}$. Slices were then washed and incubated in a secondary antibody ( $1 \mathrm{~nm}$ gold conjugated goat anti-mouse, Affiniti) diluted $1: 50$ in $20 \mathrm{mM}$ TBS pH 7.0 for 3 hours. After washing in buffer, slices were silver enhanced for 10 minutes using a commercially available kit (BioCell International Ltd), osmicated (1\% osmium tetroxide) for 1 hour and processed to epoxy resin. Negative controls involved omission of the primary antibody as for light microscopy immunocytochemistry. To overcome the variability associated with antibody access to EC within tissue slices only those vessels which opened onto the exposed vibratome face were examined as previously described. ${ }^{10}$

\section{Results}

All vessels in the negative control tissue, incubated without the primary antibody (antiEBA), were immunonegative as seen by light microscopy (Fig 1). Brain (Fig 2) and spinal cord, which were used as positive controls, showed strong EBA positive vessels in grey and white matter. Pial vessels were also well labelled.

Examination of intraocular tissues by light microscopy revealed that vessels within the optic nerve parenchyma, pial sheath of the optic nerve, and throughout the retina were strongly immunopositive for EBA (Figs 3 and 4). In the iris stroma, the majority of vessels were similarly well labelled (Fig 5). In EBA positive vessels HRP reaction product was confined to ECs and although capillaries were the most strongly labelled, arterioles and venules also expressed EBA.

Blood vessels of the ciliary body and the choroid showed a heterogeneity in EBA expression. The majority of ciliary vessels, particularly those located externally were unlabelled. However, close to the ciliary epithelium and particularly within ciliary processes, immunopositive vessels were commonly observed (Fig 5) and some immunoreactivity was apparent even at very high antibody dilutions $(1: 20000)$. The choroid showed a similar pattern of labelling. Vessels of the choriocapillaris and inner choroid were EBA positive whereas outer choroidal vessels were negative (Fig 4). EC of conjunctiva, sclera, dural sheath of optic nerve, and extraocular muscles were unlabelled (Figs 3 and 4). In the lacrimal gland, the majority of vessels were EBA negative but occasional weakly labelled vessels were observed.

Although at the light microscopy level it was observed that EBA was localised to ECs, it was not possible in these $8 \mu \mathrm{m}$ paraffin sections to define accurately its cellular location. To overcome this problem we used a pre-embedding electron microscopy immunocytochemistry method with discrete gold probes on vibratome slices of optic nerve. Examination of the luminal surface of pial and parenchymal vessels opening onto the cut faces of each slice, 


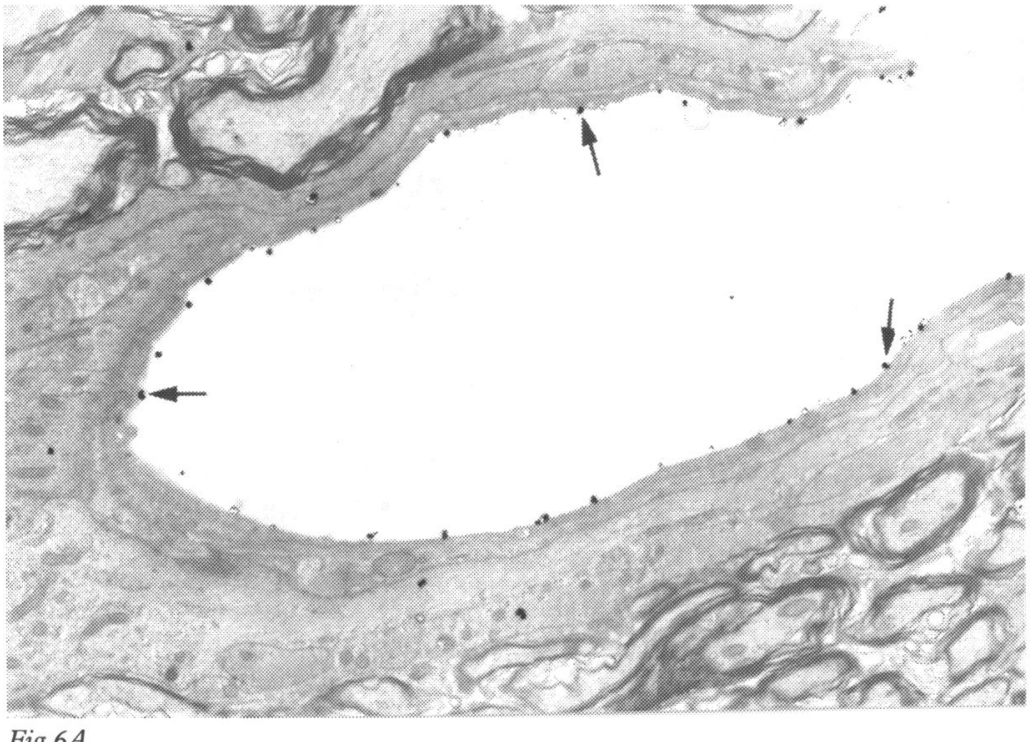

Fig $6 A$

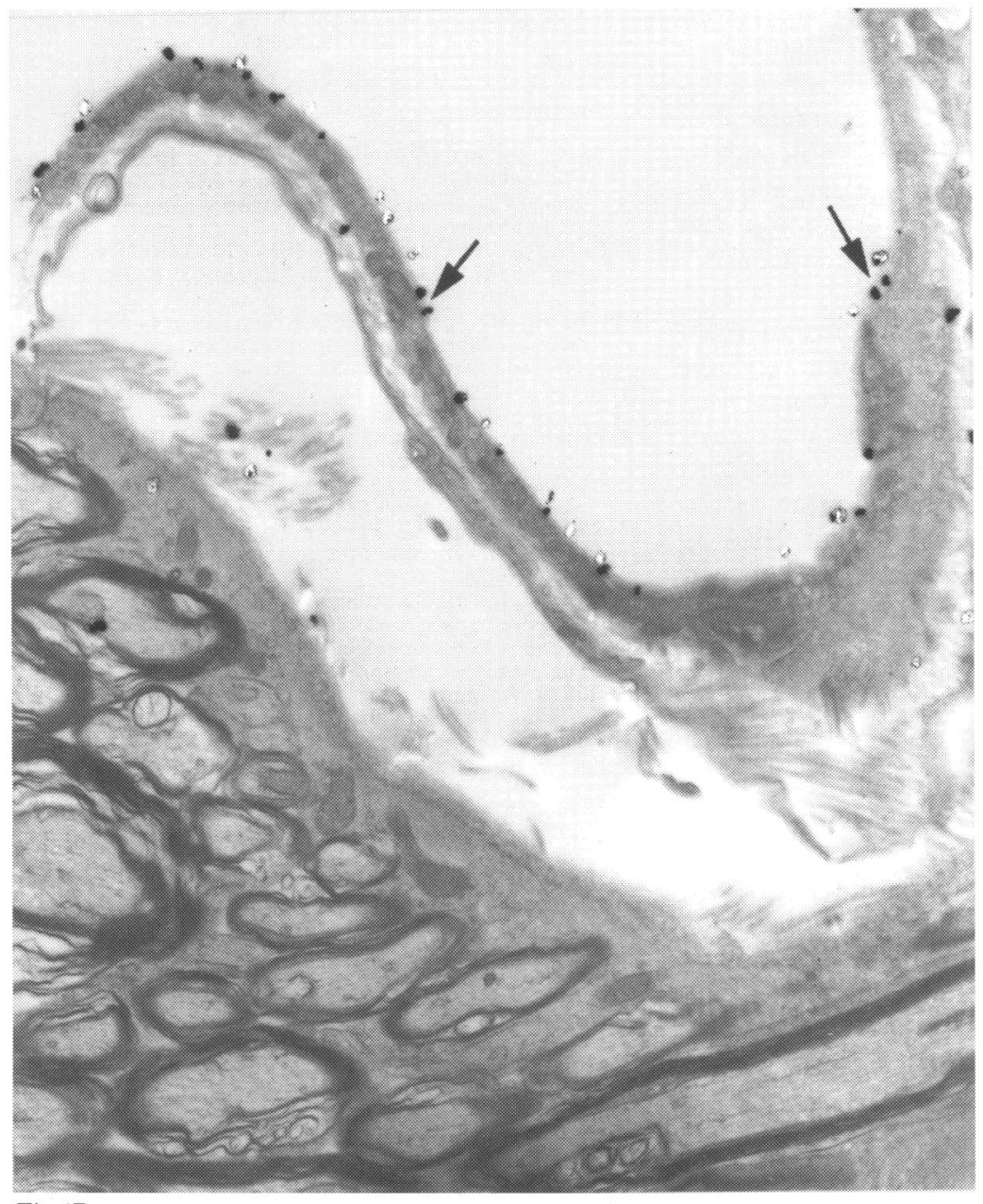

Fig $6 B$

Figure 6 Immunogold (silver enhanced) labelling of endothelial cells for endothelial barrier antigen (EBA). The label (arrows) is localised to the luminal plasma membrane. (A) Optic nerve parenchymal vessel. EBA 1:100. Magnification X4868. (B) Pial vessel. $E B A$ 1:100. Magnification $\times 10500$.

and therefore having unrestricted access to the antibodies, showed a discontinuous layer of silver enhanced immunogold particles (Fig 6). There was no apparent association with intercellular tight junctions and the label was absent from intercellular clefts. Even with the small immunogold probes $(1 \mathrm{~nm})$ used in the present study, there was relatively little tissue penetration and as a result EC cytoplasm and abluminal surface received limited exposure to anti-EBA. However, close examination of $\mathrm{EC}$ at the cut vibratome face failed to provide sufficient evidence that these regions expressed EBA.

Control electron microscopic incubations where the primary antibody was omitted were negative.

\section{Discussion}

This study demonstrated a strong expression of EBA in barrier vessels of the retina, optic nerve, pia mater, and iris. Surprisingly, several non-barrier vessels in the ciliary processes and inner part of the choroid also expressed the antigen. Vessels of the sclera, conjunctiva, dura mater, and outer vessels in the choroid and ciliary body were negative for EBA.

The retina possesses a blood-retinal barrier which shows many similarities to the BBB. ${ }^{11}$ Comparative studies of retinal and brain vessel permeability have yielded conflicting results. Lightman and coworkers ${ }^{12}$ found permeability coefficients for sucrose and mannitol which were approximately equivalent for brain and retina. In contrast the permeability of the low molecular weight tracer ${ }^{14} \mathrm{C} \alpha$ amino-isobutyric acid $\left({ }^{14} \mathrm{C}\right.$ AIB) is four times higher in retina compared with brain. ${ }^{13}$ The greater permeability of retinal EC is supported by morphological observations which show a higher number of (a) cytoplasmic vesicles and (b) shorter intercellular clefts, compared with brain EC. ${ }^{13}$ These represent possible routes through which material may traverse the vessel wall. Although retinal vessels do not express the full complement of BBB markers, several are common to both vessel types - for example, the erythrocyte/brain isoform of the glucose transporter ${ }^{14}$ and the transferrin receptor. ${ }^{15}$ The present results show that all retinal EC also express EBA.

Iridial vessels possess a significant barrier property and contribute to the blood-aqueous barrier. Studies on a variety of experimental animals have demonstrated the presence of intercellular tight junctions which restrict the passage of protein tracers such as horseradish peroxidase (HRP) ${ }^{1}$ In the rat the permeability of radiolabelled AIB was recently found to be similar for vessels of iris and retina. ${ }^{13}$ In the present study iris vessels were consistently well labelled with EBA. Interestingly, previous studies have shown that iridial vessels express a number of other barrier markers - for example, the glucose transporter ${ }^{14}$ and the membrane P-glycoprotein, ${ }^{15}$ which is known to localise to blood-tissue barriers.

Within the ciliary body and choroid EC showed a heterogeneity in EBA expression. However, the general pattern of labelling between the two tissues was similar. In the ciliary body, several vessels close to the epithelium and particularly within the ciliary processes were immunopositive, while more superficial vessels were negative. Similarly in the choroid, EC of the choriocapillaris and inner choroid were often labelled whereas 
those of the larger outer vessels were not. Ultrastructural studies have shown that capillaries of the choriocapillaris are fenestrated. In general, fenestrated capillaries vary significantly in their permeability to hydrophilic solutes. ${ }^{16}$ Studies of the permeability of fenestrated vessels of the choroid and ciliary body suggest that they are freely permeable to small molecules. ${ }^{1317}$ The permeability to proteins depends on the species studied. In rabbits, for example, labelled plasma proteins readily traverse the choroidal vasculature, ${ }^{17}$ whereas in the rat the extravasation of the protein tracers HRP and ferritin is limited. ${ }^{16}$ The finding of EBA immunoreactivity in fenestrated non-BOB vessels would appear to be contrary to the previous suggestion that this protein is a 'barrier antigen'. 5 There is some evidence that EBA is expressed weakly in some vessels at other non-barrier sites including the choroid plexus and spleen. ${ }^{5}$ This, together with the results of the present study, would suggest that EBA has a wider function in EC than previously recognised. Within the uveal tract EBA positive vessels were predominantly located near to the epithelial layers. The significance of this arrangement is at present unclear, and any possible inductive influence by these epithelial cells of neuroectodermal origin on EBA expression is speculative.

Several barrier endothelial specific markers have been described, including tight junction associated proteins and specific transporter molecules. ${ }^{34}$ In view of its expression in leaky choroidal and ciliary vessels EBA is unlikely to belong to either category. Its localisation on the EC luminal front may suggest that EBA is involved in the adhesive interaction between the EC and inflammatory cells. Several cell adhesion molecules (CAMs) have been demonstrated on chorioretinal ECs - for example, ICAM-1 and E-selectin (ELAM-1). ${ }^{18}$ These CAMs are upregulated in inflammatory conditions such as experimental autoimmune uveoretinitis (EAU) ${ }^{19}$ which is characterised by increased leucocyte adhesion and migration across the chorioretinal vasculature. Since the pathogenesis of EAU is similar to the inflammatory brain disease $\mathrm{EAE}^{19}$ the finding of a downregulation of $\mathrm{EBA}$ in the active phase of $\mathrm{EAE}^{7}$ makes this interpretation of an adhesive function for EBA open to question.

In conclusion, EBA does not have an exclusive association with barrier EC in the eye. The properties of EBA remain unknown and therefore further studies on EBA expression in normal and pathological tissues are required to elucidate its function.

We thank Roger Ward, Nescott, Ewell, Surrey for production of the colour micrographs. The work is funded by the Medical Research Council, UK.

1 Raviola G. The structural basis of the blood-ocular barriers Exp Eye Res (suppl) 1977; 27-63.

2 Bradbury MWB. The blood-brain barrier. Exp Physiol 1993; 78: 453-72.

3 Schlosshauer B. The blood-brain barrier: morphology, molecules and neurothelin. BioEssays 1993; 15: 341-6.

4 Dermietzel R, Krause D. Molecular anatomy of the bloodbrain barrier as defined by immunocytochemistry. Int Rev Cytol 1991; 127: 57-109.

5 Sternberger NH, Sternberger LA. Blood-brain barrier protein recognized by monoclonal antibody. Proc Nat Acad Sci USA 1987; 84: 8169-73.

6 Rosenstein JM, Krum JM, Sternberger LA, Pulley MT Sternberger NH. Immunocytochemical expression of the endothelial barrier antigen (EBA) during brain angiogenesis. Dev Brain Res 1992; 66: 47-54.

7 Sternberger NH, Sternberger LA, Kies MW, Shear CR. Cell surface endothelial proteins altered in experimental allergic encephalomyelitis. F Neuroimmunol 1989; 21: 241-8.

8 Mooradian AD, Grabau G, Uko-eninn A. In situ quantitative estimates of the age-related and diabetes-related changes in cerebral endothelial barrier antigen. Brain Res 1993; 609: 41-4.

9 Lawrenson JG, Ghabriel MN, Reid AR, Gajree TN, Allt G. Differential expression of an endothelial barrier antigen between the CNS and the PNS. F Anat 1995; 186: 217-21.

10 Ghabriel MN, Lawrenson JG, Reid AR, Allt G. Endothelial anionic surface charge: the effect of its neutralization on the blood nerve barrier. Endothelium 1994; 2: 239-48.

11 Bradbury MWB, Lightman SL. The blood-brain interface. Eye 1990; 4: 249-54.

12 Lightman SL, Palestine AG, Rapoport SI, Rechthand E. Quantitative assessment of the permeability of the rat blood-retinal barrier to small water-soluble nonelectrolytes. F Physiol 1987; 389: 483-90.

13 Stewart PA, Tuor UI. Blood-eye barriers in the rat: correlation of ultrastructure with function. $\mathcal{F}$ Comp Neurol 1994 340: 566-76.

14 Harik SI, Kalaria RN, Whitney PM, Andersson L, Lundah $P$, Ledbetter $S R$, et al. Glucose transporters are abundan in cells with occluding junctions at the blood-eye barriers. Proc Natl Acad Sci USA 1990; 87: 4261-4.

15 Holash JA, Stewart PA. The relationship of astrocyte-like cells to the vessels that contribute to the blood-ocular cells to the vessels that contribute to
barriers. Brain Res 1993; 629: 218-24.

16 Pino RM. Cytochemical studies of the vascular endothelium. Prog Histochem Cytochem 1989; 19: 1-37.

17 Tornquist P, Alm A, Bill A. Permeability of ocular vessels and transport across the blood-retinal barrier. Eye 1990, 4: 303-9.

18 Duguid IGM, Boyd AW, Mandel TE. Adhesion molecules are expressed in the human retina and choroid. Curr Eye Res 1992; 11 (suppl): 153-9.

19 Greenwood J. The blood-retinal barrier in experimenta autoimmune uveoretinitis (EAU): a review. Curr Eye Res 1992; 11 (suppl): 25-32. 